\title{
Forgetting literals with varying propositional symbols
}

\author{
Yves Moinard \\ IRISA, Campus de Beaulieu, 35042 RENNES-Cedex FRANCE \\ moinard@irisa.fr
}

\begin{abstract}
Recently, the old logical notion of forgetting propositional symbols (or reducing the logical vocabulary) has been generalized to a new notion: forgetting literals. The aim was to help the automatic computation of various formalisms which are currently used in knowledge representation. We extend here this notion, by allowing propositional symbols to vary while forgetting literals. The definitions are not really more complex than for literal forgetting without variation. We describe the new notion, on the syntactical and the semantical side. Then, we show how to apply it to the computation of circumscription. This computation has been done before with standard literal forgetting, but here we show how introducing varying propositional symbols simplifies significantly the computation. We revisit a fifteen years old result about computing circumscription, showing that it can be improved in the same way. We provide hints in order to apply this forgetting method also to other logical formalisms.
\end{abstract}

\section{Introduction}

The well-known notion of forgetting propositional symbols has been used for a long time in mathematical logic and in its applications for knowledge representation. Recently, [Lang et al., 2003] has extended this notion in a significant manner, by allowing the forgetting of literals. The main purpose of the authors was the introduction of a new way for helping the automatic computation of various formalisms.

The example that they provided concerns circumscription.

We extend the notion by allowing some propositional symbols to vary when forgetting literals. The new definitions are a simple and natural extension of the original ones, and they have the same kind of behavior.

We show that with this simple extension, the result given in [Lang et al., 2003] for circumscription, which involves a two stages operation, can be reduced into a single stage. We extend the correlation (given in [Lang et al., 2003]) of the method with an earlier proposal for computing circumscription [Przymusinski, 1989]. Precisely, we show that Przymun- sinski's method, which is a two stages method also, can similarly be reduced into a single stage method.

Meanwhile, we show that this way of using the notion of literal forgetting is a particular case of a powerful formalism, introduced in [Siegel and Forget, 1996] in order to facilitate the computation for nonmonotonic reasoning.

\section{Technical preliminaries}

We work in a propositional language PL. As usual, PL also denotes the set of all the formulas, and the vocabulary of $\mathbf{P L}$ is a set of propositional symbols denoted by $\mathcal{V}(\mathbf{P L})$. We restrict our attention to finite sets $\mathcal{V}(\mathbf{P L})$ in this text.

Letters $\varphi, \psi$ denote formulas in PL. Two logical constants $\top$ and $\perp$ denote respectively the true and the false formulas.

Letters $\omega, \mu, \nu$ denote interpretations for $\mathbf{P L}$, identified with subsets of $\mathcal{V}(\mathbf{P L})$. The notations $\omega \models \varphi$ and $\omega \models X$ for a set $X$ of formulas are defined classically.

For a set $E, \mathcal{P}(E)$ denotes the set of the subsets of $E$. The set $\mathcal{P}(\mathcal{V}(\mathbf{P L}))$ of the interpretations for $\mathbf{P L}$ is denoted by Mod. A model of $X$ is an interpretation $\omega$ such that $\omega \models X$, $\operatorname{Mod}(\varphi)$ and $\operatorname{Mod}(X)$ denote respectively the sets of the models of $\{\varphi\}$ and $X$.

A literal $l$ is either a symbol $p$ in $\mathcal{V}(\mathbf{P L})$ (positive literal) or its negation $\neg p$ (negative literal). A clause (respectively a term) is a disjunction (respectively a conjunction) of literals. Subsets of $\mathcal{V}(\mathbf{P L})$ are denoted by $P, Q, V$.

$P^{+}$(respectively $P^{-}$) denotes the set of the positive (respectively negative) literals built on $P$, and $P^{ \pm}$denotes the set $P^{+} \cup P^{-}$of all the literals built on $P\left(P\right.$ and $P^{+}$can often be assimilated).

For any (finite) set $X$ of formulas, $\bigwedge X$ (respectively $\bigvee X$ ) denotes the conjunction (respectively disjunction) of all the formulas in $X$. We get: $\bigwedge X \equiv X, \bigwedge \emptyset \equiv \top$ and $\bigvee \emptyset \equiv \perp$.

$\mathcal{V}(X)$ denotes the set of the propositional symbols appearing in $X$.

A disjunctive normal form or DNF (respectively conjunctive normal form or CNF) of $\varphi$ is a disjunction of consistent terms (respectively a conjunction of non trivial clauses) which is equivalent to $\varphi$. 
A prime $C N F$ is a $\mathrm{CNF}$ which involves only prime implicates: for any clauses $c, c_{i}$, if $\varphi \models c$, and $c \models c_{i}$ for some $c_{i}$ in the CNF, then $c_{i} \models c$.

A set $L$ of literals in $V^{ \pm}$(and the term $\bigwedge L$ ) is consistent and complete in $V$ if each propositional symbol of $V$ appears once and only once in $L$; the clause $\bigvee L$ is then non trivial and complete in $V$. For any set $L$ of literals, $\neg L$ denotes the set of the literals complementary to those in $L$.

We need the following notions and notations, many of them coming from [Lang et al., 2003]:

If $\varphi$ is some formula and $p$ is a propositional symbol in $\mathbf{P L}$, then $\varphi_{p \leftarrow 1}$ (respectively $\varphi_{p \leftarrow 0}$ ) is the formula obtained from $\varphi$ by replacing each occurrence of $p$ by $\top$ (respectively $\perp$ ). If $l$ is the positive literal $p$ (respectively the negative literal $\neg p$ ), then $\varphi_{l \leftarrow i}$ denotes the formula $\varphi_{p \leftarrow i}$ (respectively $\varphi_{p \leftarrow(1-i)}$ ), for $i \in\{0,1\}$.

Notations 2.1 1. If $v_{1}, \cdots, v_{n}$ are propositional symbols, $\varphi\left(v_{1} \leftarrow i_{1}, \cdots, v_{n} \leftarrow i_{n}\right)$ with each $i_{j} \in\{0,1\}$ denotes the formula $\left(\cdots\left(\left(\varphi_{v_{1} \leftarrow i_{1}}\right)_{v_{2} \leftarrow i_{2}}\right) \cdots\right)_{v_{n} \leftarrow i_{n}}$.

If the $v_{j}$ 's in the list are all distinct, then the order of the $v_{j}$ 's is without consequence for the final result. Thus, if $V_{1}$ and $V_{2}$ are disjoint subsets of $V$, we may define

$\varphi_{\left[V_{1} \leftarrow 1, V_{2} \leftarrow 0\right]}$ as

$\varphi_{\left[v_{1} \leftarrow 1, \cdots, v_{n} \leftarrow 1, v_{n+1} \leftarrow 0, \cdots, v_{n+m} \leftarrow 0\right]}$, where $\left(v_{1}, \cdots, v_{n}\right)$ and $\left(v_{n+1}, \cdots, v_{n+m}\right)$ are two orderings of all the elements of $V_{1}$ and $V_{2}$ respectively.

2. If $L=\left(l_{1}, \cdots, l_{n}\right)$ is a list of literals, then

$\varphi\left(l_{1} \leftarrow i_{1} \cdots l_{n} \leftarrow i_{n}\right)$ denotes the formula

$\left(\cdots\left(\left(\varphi_{l_{1} \leftarrow i_{1}}\right) l_{2} \leftarrow i_{2}\right) \cdots\right)_{l_{n} \leftarrow i_{n}}$.

3. Let $\mathcal{V}(\mathbf{P L})^{ \pm}$be ordered in some arbitrary way. If $L_{1}, \cdots, L_{n}$ are disjoint sets of literals,

$\varphi\left\langle L_{1} \leftarrow i_{1}, \cdots, L_{n} \leftarrow i_{n}\right\rangle$ denotes the formula

$\varphi\left(l_{1} \leftarrow k_{1}, \cdots, l_{n} \leftarrow k_{n}\right)$ where $\left(l_{1}, \cdots, l_{n}\right)$ is the enumeration of the set $L_{1} \cup \cdots \cup L_{n}$ which respects the order chosen for the set of all the literals, and where, for each $l_{j}, k_{j}$ is equal to $i_{r}$ where $r \in\{1, \cdots, n\}$ is such that $l_{j} \in L_{r}$.

Let us remind a well known notion, variable forgetting:

Definition 2.2 If $V \subseteq \mathcal{V}(\mathbf{P L})$ and $\varphi \in \mathbf{P L}$, Forget $V(\varphi, V)$ denotes a formula, in the propositional language $\mathbf{P L} \bar{V}$ built on the vocabulary $\bar{V}=\mathcal{V}(\mathbf{P L})-V$, which is equivalent to $\varphi$ in this restricted language: Forget $V(\varphi, V) \equiv T h(\varphi) \cap \mathbf{P} \mathbf{L}_{\bar{V}}$ where $\operatorname{Th}(\varphi)=\left\{\varphi^{\prime} \in \mathbf{P L} / \varphi \models \varphi^{\prime}\right\}$.

For any $\psi \in \mathbf{P L}_{\bar{V}}, \varphi \models \psi$ iff $\operatorname{Forget} V(\varphi, V) \models \psi$.

Various ways are known in order to get $\operatorname{Forget} V(\varphi, V)$, the two easiest (to describe) ways being the following ones:

1. In a DNF form of $\varphi$, suppress all the literals in $V^{ \pm}$.
2. In a prime $\mathrm{CNF}$ form of $\varphi$, suppress any clause containing a symbol in $V$.

Remark 2.3 When considering a formula equivalent to a set $T h(\varphi) \cap X$, the set of formulas $X$ can be replaced by any set $Y$ having the same $\wedge$-closure: $\left\{\bigwedge X^{\prime} / X^{\prime} \subseteq X\right\}=$ $\left\{\bigwedge X^{\prime} / X^{\prime} \subseteq Y\right\}$. Indeed, we have:

- If $X$ and $Y$ have the same $\wedge$-closure, then $T h(\varphi) \cap X \equiv$ $T h(\varphi) \cap Y$.

- The converse is true, provided that we assimilate equivalent formulas: if $T h(\varphi) \cap X \equiv T h(\varphi) \cap Y$ for any $\varphi \in \mathbf{P L}$, then $X$ and $Y$ have the same $\wedge$-closure.

Since we work in finite propositional languages, there exists a unique smallest (for set inclusion, and up to logical equivalence) possible set, the $\wedge$-reduct of $X$, equal to the set $X-\{\varphi \in X / \varphi$ is in the $\wedge$-closure of $X-\{\varphi\}\}$.

Thus, $X$ can be replaced by any set which contains the $\wedge$-reduct of $X$ and is included in the $\wedge$-closure of $X$.

Thus, instead of considering the whole set $\mathbf{P} \mathbf{L}_{\bar{V}}$ in Forget $V(\varphi, V) \equiv T h(\varphi) \cap \mathbf{P L}_{\bar{V}}$ (Definition 2.2), we can consider the set of all the clauses built on $\bar{V}$, the smallest (for $\subseteq$ ) set that can be considered here being the set of these clauses which are non trivial and complete in $\bar{V}$.

From a semantical side, the set of the models of Forget $V(\varphi, V)$ is the set of all the interpretations for $\mathbf{P L}$ which coincide with a model of $\varphi$ for all the propositional symbols not in $V$.

\section{Literal forgetting}

Variable forgetting as been generalized as detailed now, beginning by the semantical side.

Definition 3.1 [Lang et al., 2003, pp. 396-397] Let $\omega$ be an interpretation for $\mathbf{P L}$, $p$ be a propositional symbol in $\mathbf{P L}$ and $L$ be a consistent set of literals in $\mathrm{PL}$.

We define the interpretations

$\operatorname{Force}(\omega, p)=\omega \cup\{p\}$ and $\operatorname{Force}(\omega, \neg p)=\omega-\{p\}$ and more generally,

$\operatorname{Force}(\omega, L)=$

$\omega \cup\{p / p \in \mathcal{V}(\mathbf{P L}), p \in L\}-\{p / p \in \mathcal{V}(\mathbf{P L}), \neg p \in L\}$.

Thus, $\operatorname{Force}(\omega, L)$ is the interpretation for $\mathbf{P L}$ which is equal to $\omega$ for all the propositional symbols in $\mathcal{V}(\mathbf{P L})-\mathcal{V}(L)$ and which satisfies all the literals of $L$.

Definition 3.2 (Literal forgetting) [Lang et al., 2003, Prop. 15] If $\varphi$ is a formula and $L$ a set of literals in PL, ForgetLit $(\varphi, L)$ is a formula having for models the set of all the interpretations for $\mathrm{PL}$ which can be turned into a model of $\varphi$ when forced by a consistent subset of $L$ :

$\operatorname{Mod}(\operatorname{Forget} \operatorname{Lit}(\varphi, L))=\{\omega /$

Force $\left(\omega, L_{1}\right) \models \varphi$ and $L_{1}$ is a consistent subset of $\left.L\right\}$. 
This amounts to say that the models of $\operatorname{Forget} \operatorname{Lit}(\varphi, L)$ are built from the models of $\varphi$ by allowing to negate an arbitrary number of values of literals in $L$ :

$$
\begin{aligned}
& \operatorname{Mod}(\text { ForgetLit }(\varphi, L))=\left\{\text { Force }\left(\omega^{\prime}, L_{1}^{\prime}\right) /\right. \\
& \left.\omega^{\prime} \models \varphi \quad \text { and } L_{1}^{\prime} \text { is a consistent subset of } \neg L\right\}
\end{aligned}
$$

Let us consider the syntactical side now. One way is to start from a DNF formulation of $\varphi$ :

Proposition 3.3 [Lang et al., 2003] If $\varphi=t_{1} \vee \cdots \vee t_{n}$ is a DNF, then ForgetLit $(\varphi, L)$ is equivalent to the formula $t_{1}^{\prime} \vee \cdots \vee t_{n}^{\prime}$ where $t_{i}^{\prime}$ is the term $t_{i}$ without the literals in $L$.

The similar method for obtaining $\operatorname{Forget} V(\varphi, V)$ when $\varphi$ is a DNF has been reminded in point 1 following Definition 2.2. Remind also that, for any $\varphi, \operatorname{Forget} V(\varphi, V)$ can be defined as follows (cf Notations 2.1-1):

$$
\operatorname{Forget} V(\varphi, V)=\bigvee_{V^{\prime} \subseteq V} \varphi_{\left[V^{\prime} \leftarrow 1, \quad\left(V-V^{\prime}\right) \leftarrow 0\right]} .
$$

Similarly, the following syntactical definition can be given:

Definition 3.4 If $L$ is a set of literals in $\mathbf{P L}$, then

$$
\text { ForgetLit }(\varphi, L)=\bigvee_{L^{\prime} \subseteq L}\left(\left(\bigwedge \neg L^{\prime}\right) \wedge \varphi_{\left.\left\langle\left(L-L^{\prime}\right) \leftarrow 1\right\rangle\right)}\right. \text {. }
$$

This is a paraphrase of [Lang et al., 2003, Definition 7]. We refer the reader to this text which shows

1. the adequacy with Definition 3.2 and Proposition 3.3,

2. that choosing any order of the literals does not modify the meaning of the final formula (cf Notations 2.1-3), and

3. that we get also

$\operatorname{Forget} \operatorname{Lit}(\varphi, L) \equiv \bigvee_{L^{\prime} \subseteq L}\left(\left(\bigwedge \neg L^{\prime}\right) \wedge \varphi_{\left\langle\left(L-L^{\prime}\right) \leftarrow 1, L^{\prime} \leftarrow 0\right\rangle}\right)$.

The presence of $\left(\bigwedge_{l^{\prime} \in L^{\prime}} \neg l^{\prime}\right)$, which is what differentiates Forget $\operatorname{Lit}(\varphi, \ldots)$ from $\operatorname{Forget} V(\varphi, \ldots)$, comes from the fact that here we want to forget $l \in L$, but not $l^{\prime} \in \neg L$.

A proof in [Lang et al., 2003], using Proposition 3.3, shows that we get $\operatorname{Forget} \operatorname{Lit}\left(\varphi, V^{ \pm}\right) \equiv \operatorname{Forget} V(\varphi, V)$.

This proof is easily extended to get:

Remark 3.5 Since any set of literals can be written as a disjoint union between a consistent set $L^{\prime}$ and a set $V^{ \pm}$of complementary literals, here is a useful formulation:

ForgetLit $\left(\varphi, L^{\prime} \cup V^{ \pm}\right) \equiv$ ForgetLit $\left(\right.$ Forget $\left.V(\varphi, V), L^{\prime}\right)$.

(Notice that $L^{\prime}$ can even be inconsistent in this equivalence.)

This remark has the advantage of separating clearly the literals which are forgotten and the propositional symbols which are forgotten. Let $V^{\prime}$ denote the set $\mathcal{V}\left(L^{\prime}\right)$ of the propositional symbols in $L^{\prime}$, and $V^{\prime \prime}=\mathcal{V}(\mathbf{P L})-V-V^{\prime}$ be the set of the remaining symbols. Then we get:
1. The propositional symbols in $V$ are forgotten;

2. the literals in $L^{\prime}$ are forgotten, but their symbols (in $V^{\prime}$ ) may remain, since the literals in $\neg L^{\prime}$ are not forgotten;

3. the literals in $V^{\prime \prime \pm}$ are not forgotten, thus the propositional symbols in $V^{\prime \prime}$ are fixed.

Thus, ForgetLit $\left(\varphi, L_{1}\right)$ can be defined as: forgetting literals with some propositional symbols fixed. It is tempting to generalize the notion, by allowing some propositional symbols to vary in the forgetting process.

\section{Literal forgetting with varying symbols}

As done with the original notion, it is convenient to introduce the semantical definitions first.

Definition 4.1 Let $\varphi$ be a formula, $V$ a set of propositional symbols, and $L$ a consistent set of literals, in $\mathbf{P L}$, with $V$ and $\mathcal{V}(L)$ disjoint in $\mathcal{V}(\mathbf{P L})$. ForgetLitVar $(\varphi, L, V)$ is a formula having the following set of models:

$$
\begin{aligned}
& \operatorname{Mod}(\text { ForgetLitVar }(\varphi, L, V))=\{\omega / \\
& \operatorname{Force}\left(\omega, L_{1} \cup L_{2}\right) \models \varphi \text { where } L_{1} \subseteq L, L_{2} \subseteq V^{ \pm}, \\
& \left.L_{2} \text { consistent, and }\left(\omega \not L_{1} \text { or } L_{2}=\emptyset\right)\right\} .
\end{aligned}
$$

This is equivalent to:

$$
\begin{aligned}
& \operatorname{Mod}(\text { ForgetLitVar }(\varphi, L, V))= \\
& \left\{\text { Force }\left(\omega, L_{1} \cup L_{2}\right) / \omega \models \varphi\right. \text { where } \\
& \quad L_{1} \subseteq \neg L, L_{2} \subseteq V^{ \pm}, \quad L_{2} \text { consistent and } \\
& \left.\quad\left(\omega \not \models L_{1} \text { or } L_{2}=\emptyset\right)\right\} .
\end{aligned}
$$

Since $\omega \models L_{2}$ iff $\operatorname{Force}\left(\omega, L_{2}\right)=\omega$, in these two formulations the condition " $\left(\omega \not \models L_{1}\right.$ or $\left.L_{2}=\emptyset\right)$ " can be replaced by " $\left(\omega \not \models L_{1}\right.$ or $\left.\omega \models L_{2}\right)$ ", and then we can replace " $L_{2}$ consistent" by " $L_{2}$ consistent and complete in $V$ ".

We could be more general, by allowing to forget some propositional symbols, which amounts to allow non consistent sets $L$. This generalization does not present difficulties, however, since we have not found any application for it till now, we leave it for future work.

With respect to Definition 3.2, what happens here is that the non consistent part of the set of literals, which allowed to forget some set $V$ of propositional symbols altogether, has been replaced by a set of varying propositional symbols.

Remark 4.2 Since ForgetLit $\left(L_{1}, \varphi\right) \models$ ForgetLit $\left(L_{1} \cup\right.$ $\left.L_{2}, \varphi\right)$ holds from [Lang et al., 2003], we get:

$$
\varphi \models \operatorname{Forget} V(\varphi, V) \models \operatorname{Forget} L i t\left(\varphi, L \cup V^{ \pm}\right) \text {. }
$$

It is immediate to show that we get also:

$$
\varphi \models F \operatorname{orget} L i t \operatorname{Var}(\varphi, L, V) \models \operatorname{Forget} L i t\left(\varphi, L \cup V^{ \pm}\right) \text {. }
$$

Here are the motivations for introducing ForgetLitVar: we want to "forget" the literals in $L$, even at the price of modifying the literals in $V^{ \pm}$. This is what explains that, if we effectively forget at least one literal in $L$, then, we allow 
any modification for the literals in $V^{ \pm}$. However, we do not want to modify the literals in $V^{ \pm}$"for nothing": our aim is to forget as many literals in $L$ as possible. This justifies the condition " $\left(\omega \not \forall L_{1}\right.$ or $\left.L_{2}=\emptyset\right)$ " in the definition.

The syntactical aspect is slightly more tricky, but it remains rather simple and it allows to revisit and improve already known results. As with the original notion (see Proposition 3.3), the simplest way is to start from a DNF.

Without loss of generality we can consider that $L$ is a set of negative literals (otherwise, replace any $p \in \mathcal{V}(L)$ such that $p \in L$ by $\neg p^{\prime}, p^{\prime}$ being a new propositional symbol, then after the computations, replace $p^{\prime}$ by $\neg p$ ). Thus, till now, we will consider two disjoint subsets $P$ and $V$ of $\mathcal{V}(\mathbf{P L})$, and $L=\neg P$ with $Q=\mathcal{V}(\mathbf{P L})-V-P$ denoting the set of the remaining propositional symbols.

Proposition 4.3 Let $\varphi=t_{1} \vee \cdots \vee t_{n}$ be a DNF, with

$$
t_{i}=\left(\bigwedge P_{i, 1}\right) \wedge\left(\bigwedge \neg\left(P_{i, 2}\right)\right) \wedge\left(\bigwedge V_{i, l}\right) \wedge\left(\bigwedge Q_{i, l}\right),
$$

where $P_{i, 1} \subseteq P, P_{i, 2} \subseteq P-P_{i, 1}$, and where $V_{i, l}$ and $Q_{i, l}$ are consistent sets of literals in $V^{ \pm}$and $Q^{ \pm}$respectively. Then ForgetLitVar $\left(\varphi, P^{-}, V\right) \equiv t_{1}^{\prime} \vee \cdots \vee t_{n}^{\prime}$ where

$$
\begin{gathered}
t_{i}^{\prime}=\left(\bigwedge P_{i, 1}\right) \wedge\left(\bigwedge Q_{i, l}\right) \wedge\left(\left(\bigvee\left(P-P_{i, 1}\right)\right) \vee\left(\bigwedge V_{i, l}\right)\right), i . e . \\
t_{i}^{\prime}=\left(\bigwedge P_{i, 1}\right) \wedge\left(\bigwedge Q_{i, l}\right) \wedge \bigwedge_{l \in V_{i, l}}\left(l \vee\left(\bigvee\left(P-P_{i, 1}\right)\right)\right) .
\end{gathered}
$$

Thus, $t_{i}^{\prime}$ is $t_{i}$ except that the literals in $\neg P$ are suppressed while each literal in $V^{ \pm}$must be disjuncted with the clause $\bigvee\left(P-P_{1}\right)$, this clause denoting the disjunction of all the literals in $P^{+}$which do not appear (positively) in $t_{i}$.

Proof: Let us consider complete terms first, such as

$$
t_{i}=t=\left(\bigwedge P_{1}\right) \wedge\left(\bigwedge \neg\left(P-P_{1}\right)\right) \wedge\left(\bigwedge V_{l}\right) \wedge\left(\bigwedge Q_{l}\right),
$$

where $P_{1} \subseteq P, V_{l}$ and $Q_{l}$ being consistent and complete sets of literals in $V$ and $Q$ respectively. $t$ corresponds to an interpretation $\omega$. The set $F(\omega)=$

$\left\{F \operatorname{Frce}\left(\omega, L_{1} \cup L_{2}\right) / L_{1} \subseteq P, L_{2} \subseteq V^{ \pm}\right.$,

$L_{2}$ consistent and complete in $V$, and $\omega \not \models L_{1}$ or $\left.\omega \models L_{2}\right\}$ is the set of the models of the formula $t^{1} \wedge t^{2}$ where $t^{1}=\left(\bigwedge P_{1}\right) \wedge\left(\bigwedge Q_{l}\right)$ and $\left.t^{2}=\left(\bigvee\left(P-P_{1}\right)\right) \vee\left(\bigwedge V_{l}\right)\right)$

Indeed, for each $\omega^{\prime} \in F(\omega), t^{1}$ holds since it holds in $\omega$, and the symbols in $P-P_{1}$ and $V$ can take any value satisfying the condition $\omega \not \models L_{1}$ or $\omega \models L_{2}$. Since $\omega \models t$, this means $L_{1} \cap\left(P-P_{1}\right) \neq \emptyset$ or $L_{2} \subseteq V_{l}$, which is equivalent to $\omega^{\prime} \models t_{2}$. Conversely, any model $\omega^{\prime \prime}$ of $t_{1} \wedge t_{2}$ is easily seen to be in $F(\omega)$.

The same result holds for any (consistent) term $t=t_{i}=$ $\left(\bigwedge P_{1}\right) \wedge\left(\bigwedge \neg\left(P_{2}\right)\right) \wedge\left(\bigwedge V_{l}\right) \wedge\left(\bigwedge Q_{l}\right)$, where $P_{1} \subseteq P$, $P_{2} \subseteq P-P_{1}, V_{l}$ and $Q_{l}$ being consistent subsets of $V^{ \pm}$and $Q^{ \pm}$respectively: Let us first consider separately the cases where some symbols in $P$ are missing, then symbols in $V$, then symbols in $Q$.
(1) If $p \in P$ is missing in $t$ ( $p$ and $\neg p$ are missing), for any model $\omega^{\prime}$ of $t, \omega^{\prime \prime}=\operatorname{Force}\left(\omega^{\prime},\{\neg p\}\right)$ and $\operatorname{Force}\left(\omega^{\prime \prime},\{p\}\right)$ are two models of $t$ (one of these is $\omega^{\prime}$ ). By considering all the missing $p$ 's, we get that the set

$\left\{F \operatorname{Frce}\left(\omega^{\prime}, L_{1} \cup L_{2}\right) / \omega^{\prime} \models t, \quad L_{1} \subseteq \neg P, L_{2}\right.$ consistent $\subseteq$ $V^{ \pm}, \omega^{\prime} \not \neq L_{1}$ or $\left.L_{2}=\emptyset\right\} \quad$ is included in the set $\left\{F \operatorname{Force}\left(\omega^{\prime \prime}, L_{1} \cup L_{2}\right) \quad / \quad \omega^{\prime \prime} \models t \wedge \bigwedge \neg\left(P-P_{1}\right), L_{1} \subseteq\right.$ $\neg P, L_{2}$ consistent $\subseteq V^{ \pm}, \omega^{\prime \prime} \not \neq L_{1}$ or $\left.L_{2}=\emptyset\right\}$.

Thus any missing $p$ in $t$ behaves as if the negative literal $\neg p$ was present: we get a term "completed in $P$ " satisfying ForgetLitVar $\left(t, P^{-}, V\right) \equiv$

ForgetLitVar $\left(t \wedge \neg\left(P-P_{1}\right), P^{-}, V\right)$.

(2) The reasoning for a missing $q$ in $t(q \in Q)$ is simpler yet: if some $q \in Q$ does not appear in $t$, it can be interpreted as false or true for any model of ForgetLitVar $(t, L, Q)$, which means that we keep the part $\bigwedge Q_{l}$ unmodified, exactly as in the case where $Q_{l}$ is complete in $Q$.

(3) The case for $V$ is similar (the disjunction of all the formulas with all the possibilities for the missing symbols gives the formula where these symbols are missing): If some $v \in V$ is missing in $t$, then any model $\omega^{\prime}$ of $t$ has its counterpart where the value for $v$ is modified. Let us call $V_{m}$ the set of the symbols in $V$ which are absent in $t$. By considering the disjunctions of all the possibilities, we get the formula $\bigvee_{V_{l}^{\prime} \in \mathcal{L}_{m}}\left(\left(\bigwedge P_{1}\right) \wedge\left(\bigwedge Q_{l}\right) \wedge\left(\left(\bigvee\left(P-P_{1}\right)\right) \vee\left(\bigwedge V_{l} \wedge \wedge V_{l}^{\prime}\right)\right)\right)$ where $\mathcal{L}_{m}$ is the set of all the sets of literals consistent and complete in $V_{m}$. This is equivalent to the formula $\left(\bigwedge P_{1}\right) \wedge\left(\bigwedge Q_{l}\right) \wedge\left(\left(\bigvee\left(P-P_{1}\right)\right) \vee\left(\bigwedge V_{l}\right)\right)$

Combining "the three incompleteness" (1)-(3) gives:

ForgetLitVar $\left(t_{i}, P^{-}, V\right) \equiv$

$\left(\bigwedge P_{1}\right) \wedge\left(\bigwedge Q_{l}\right) \wedge\left(\left(\bigvee\left(P-P_{1}\right)\right) \vee\left(\bigwedge V_{l}\right)\right)$

The disjunction for all the $t_{i}$ 's gives the result.

We have provided the semantical definition (in the lines of Definition 3.2) and a characterization from a DNF formulation (in the lines of Proposition 3.3). Let us provide now other characterizations, and a comparison with Forget Lit.

Proposition 4.4 Let $\varphi$ be a formula in $\mathrm{PL}$, and $P, Q$ and $V$ be three pairwise disjoint sets of propositional symbols such that $P \cup Q \cup V=\mathcal{V}(\mathbf{P L})$.

1. ForgetLit $\left(\varphi, P^{-} \cup V^{ \pm}\right)$is equivalent to the set $\operatorname{Th}(\varphi) \cap X$ where $X$ is the set of all the formulas in $\mathbf{P L}$ which are disjunctions of terms of the kind $\left(\bigwedge P_{1}\right) \wedge\left(\bigwedge Q_{l}\right)$ with $P_{1} \subseteq P$ and $Q_{l} \subseteq Q^{ \pm}$ (we can clearly consider consistent sets $Q_{l}$ only).

2. ForgetLitVar $\left(\varphi, P^{-}, V\right)$ is equivalent to the set $T h(\varphi) \cap X$ where $X$ is the set of all the formulas in $\mathbf{P L}$ which are disjunctions of terms of the kind $\left(\bigwedge P_{1}\right) \wedge\left(\bigwedge Q_{l}\right) \wedge \bigwedge_{l \in V_{l}}\left(l \vee\left(\bigvee\left(P-P_{1}\right)\right)\right)$, where $P_{1} \subseteq P, V_{l}$ and $Q_{l}$ being consistent sets of literals in $V^{ \pm}$and $Q^{ \pm}$respectively.

These two results are immediate consequences of Propositions 3.3 and 4.3 respectively. We get the following alternative possibilities for the sets $X$ 's, firstly by duality from the preceding results, then by considering some set 
having the same $\wedge$-closure as $X$ (Remark 2.3):

\section{Proposition 4.4 (following)}

1. (a) For ForgetLit $\left(\varphi, P^{-} \cup V^{ \pm}\right), X$ is the set of all the conjunctions of the clauses of the kind $\left(\bigvee P_{1}\right) \vee\left(\bigvee Q_{l}\right)$ with $P_{1} \subseteq P$ and $Q_{l} \subseteq Q^{ \pm}$ (we can clearly consider consistent sets $Q_{l}$ only).

(b) We can also consider the set $X$ of all the clauses $\left(\bigvee P_{1}\right) \vee\left(\bigvee Q_{l}\right)$ with $P_{1} \subseteq P$ and $Q_{l} \subseteq Q^{ \pm}$

(c) The smallest set $X$ possible is the set of all the clauses $\quad\left(\bigvee P_{1}\right) \vee\left(\bigvee Q_{l}\right)$ with $\quad P_{1} \subseteq P$, $Q_{l} \subseteq Q^{ \pm}, Q_{l}$ consistent and complete in $Q$.

2. (a) For ForgetLitVar $\left(\varphi, P^{-}, V\right), X$ is the set of all the conjunctions of the formulas flv $\left(P_{1}, Q_{l}, V_{l}\right)=$ $\left(\bigvee P_{1}\right) \vee\left(\bigvee Q_{l}\right) \vee \bigvee_{l \in V_{l}}\left(l \wedge\left(\bigwedge\left(P-P_{1}\right)\right)\right)$, where $P_{1} \subseteq P, V_{l}$ and $Q_{l}$ being consistent sets of literals in $\bar{V}^{ \pm}$and $Q^{ \pm}$respectively.

(b) We can also consider the set $X$ of all the formulas flv $\left(P_{1}, Q_{l}, V_{l}\right)$ of this kind.

(c) The smallest set $X$ possible is the set of all the formulas $f l v\left(P_{1}, Q_{l}, V_{l}\right)$ with $P_{1} \subseteq P, Q_{l}$ and $V_{l}$ being sets of literals consistent and complete in $Q$ and $\checkmark$ respectively.

These results provide the analogous, for ForgetLit and ForgetLitVar, of the results for ForgetV reminded in Definition 2.2, and in Remark 2.3.

The next definition, analogous to Definition 3.4, will be our last general result about ForgetLitVar:

Definition 4.5 If $\varphi$ is a formula and $P$ and $V$ are two disjoint subsets of $\mathcal{V}(\mathbf{P L})$, then ForgetLitVar $\left(\varphi, P^{-}, V\right)$ is the formula

$$
\begin{aligned}
& \bigvee_{P_{1} \subseteq P}\left(\bigwedge P _ { 1 } \wedge \left(\varphi_{\left[P_{1} \leftarrow 1,\left(P-P_{1}\right) \leftarrow 0\right]} \vee\right.\right. \\
& \left.\left.\quad\left(\text { Forget } V\left(\varphi_{\left[P_{1} \leftarrow 1,\left(P-P_{1}\right) \leftarrow 0\right]}, V\right) \wedge\left(\bigvee\left(P-P_{1}\right)\right)\right)\right)\right) .
\end{aligned}
$$

Proof of the adequacy with Definition 4.1: Each model $\omega$ of $\varphi$ gives rise to the following models of ForgetLitVar $\left(\varphi, P^{-}, V\right)$ :

- $\omega$ itself, model of $\psi_{1}=\bigwedge P_{1} \wedge \bigwedge \neg\left(P-P_{1}\right) \wedge$ $\varphi_{\left[P_{1} \leftarrow 1,\left(P-P_{1}\right) \leftarrow 0\right]}$ where $P_{1}=\omega \cap P, \quad$ together with

- all the interpretations differing from $\omega$ in that they have at least one more $p \in P$, and no constraint holds for the symbols in $V$; this set of interpretations being the set of models of the formula $\psi_{2}=$ $\bigwedge P_{1} \wedge$ ForgetV $\left(\varphi_{\left[P_{1} \leftarrow 1,\left(P-P_{1}\right) \leftarrow 0\right]}, V\right) \wedge \bigvee\left(P-P_{1}\right)$

Since $\varphi_{\left[P_{1} \leftarrow 1,\left(P-P_{1}\right) \leftarrow 0\right]} \models F$ orget $V\left(\varphi_{\left[P_{1} \leftarrow 1,\left(P-P_{1}\right) \leftarrow 0\right]}, V\right)$ and $\bigwedge \neg\left(P-P_{1}\right) \equiv \neg\left(\bigvee\left(P-P_{1}\right)\right)$, when considering the disjunction $\psi_{1} \vee \psi_{2}$, we can suppress $\wedge \bigwedge \neg\left(P-P_{1}\right)$ in $\psi_{1}$. The disjunction of all these formulas $\psi_{1} \vee \psi_{2}$ for each model $\omega$ of $\varphi$, gives the formula as written in this definition.

Example 1 Here $P=\{a, b\}, V=\{c\}, Q=\{d\}$, with $\varphi=(\neg a \wedge b \wedge c) \vee(a \wedge \neg b \wedge \neg c \wedge \neg d)$.
Since $\varphi$ is a DNF, the rules from a DNF in Section 2, Propositions 3.3 and 4.3 respectively for Forget $V$, Forget Lit and Forget LitVar, give the three results:

- $\operatorname{Forget} V(\varphi, V) \equiv(\neg a \wedge b) \vee(a \wedge \neg b \wedge \neg d)$.

- ForgetLit $\left(\varphi, P^{-} \cup V^{ \pm}\right) \equiv b \vee(a \wedge \neg d)$.

- ForgetLitVar $\left(\varphi, P^{-}, V\right) \equiv$

$$
(a \wedge b) \vee(a \wedge \neg c \wedge \neg d) \vee(b \wedge c) \text {. }
$$

$F L V 1$

Definitions 3.4 and 4.5 can be used also, as shown now for Definition 4.5 where, in each case, $\psi=\varphi_{\left[P_{1} \leftarrow 1,\left(P-P_{1}\right) \leftarrow 0\right]}$ :

$$
\begin{aligned}
P_{1}=\emptyset: \psi & \vee(\text { Forget } V(\psi, c) \wedge(a \vee b)) \equiv \\
\perp \vee(\perp \wedge(a \vee b)) \equiv \perp . & \\
P_{1}=\{a\}: a & \wedge(\psi \vee(F \text { orget } V(\psi, c) \wedge b)) \equiv \\
a & \wedge((\neg c \wedge \neg d) \vee(\neg d \wedge b)) . \\
P_{1}=\{b\}: b & \wedge(\psi \vee(F \text { orget } V(\psi, c) \wedge a)) \equiv \\
b & \wedge(c \vee(\top \wedge a)) . \\
P_{1}=\{a, b\}: a & \wedge b \wedge(\psi \vee(\text { Forget } V(\psi, c) \wedge \perp)) \equiv \\
a & \wedge b \wedge(\perp \vee \perp) \equiv \perp .
\end{aligned}
$$

The disjunction $\bigvee_{i=1}^{4} \varphi_{i}$ is equivalent to $F L V 1$.

Let us examine the semantical side now, starting with $\operatorname{Mod}(\varphi)=\left\{\mu_{1}, \mu_{2}, \mu_{3}\right\}$ with

$\mu_{1}=\{a\}, \mu_{2}=\{b, c\}, \mu_{3}=\{b, c, d\}$.

- The six models of Forget $V(\varphi, V)$ are obtained by adding the three interpretations differing from the three models of $\varphi$ by the value attributed to $c$ : $\mu_{1}^{\prime}=\{a, c\}, \mu_{2}^{\prime}=\{b\}, \mu_{3}^{\prime}=\{b, d\}$.

- The ten models of ForgetLit $\left(\varphi, P^{-} \cup V^{ \pm}\right)$are obtained by adding to the models of $\varphi$ the seven interpretations differing from these models by adding any subset of $\{a, b\}$ and by either do nothing else or modify the value of $c$ (adding $c$ if it is not present and removing $c$ if it is present). This gives the models of Forget $V(\varphi, V)$ plus the four interpretations including $\{a, b\}$.

- The seven models of ForgetLitVar $\left(\varphi, P^{-}, V\right)$ are obtained by adding to the models of $\varphi$ the four interpretations differing from these models by adding a non empty subset of $\{a, b\}$ and by either do nothing else or modify the value of $c$, which gives here the four interpretations including $\{a, b\}$.

Notice that on this example we get (cf Remark 4.2):

- $\operatorname{Forget} V(\varphi, V) \vee$ ForgetLitVar $\left(\varphi, P^{-}, V\right) \equiv$ ForgetLit $\left(\varphi, P^{-} \cup V^{ \pm}\right)$

- $\operatorname{Forget} V(\varphi, V) \wedge$ ForgetLitVar $\left(\varphi, P^{-}, V\right) \equiv \varphi$.

It is immediate (from the semantical characterizations) to show that these two equivalences are general.

Notice also that, once we have all the models of $\varphi$, the complexity of the construction of all the models of ForgetLitVar $\left(\varphi, P^{-}, V\right)$ is not greater than the complexity of the construction of all the models of Forget Lit $\left(\varphi, P^{-} \cup V^{ \pm}\right)$.

Let us (re-)examine an application of literal forgetting. 


\section{Circumscription and literal forgetting}

Literal forgetting has connexions with circumscription, a well known formalism in knowledge representation [McCarthy, 1986]. Circumscription minimizes "exceptions", and it is used in action languages and other formalizations of common sense reasoning. A key issue is the efficient computation, so that any step towards an amelioration can be helpful.

Definition 5.1 $\mathcal{V}(\mathbf{P L})=P \cup V \cup Q$ (disjoint sets). The propositional symbols in $P, V, Q$ are respectively circumscribed, varying, and fixed.

We define the relation $\prec_{(P, Q, V)}$ in $\mathbf{M o d}$ by

$\mu \prec_{(P, Q, V)} \nu$ if $P \cap \mu \subset P \cap \nu$ and $Q \cap \mu=Q \cap \nu$

(strict $\subset$, no condition for $V$ ).

A model $\mu$ of $\varphi$ is minimal for $\prec_{(P, Q, V)}$ if for no model $\nu$ of $\varphi$ we have $\nu \prec \mu$.

The circumscription $\operatorname{CIRC}(P, Q, V)$ is the mapping $\mathbf{P L} \hookrightarrow \mathcal{P}(\mathbf{P L})$ defined as follows:

for any $\varphi, \psi$ in $\mathbf{P L}, \psi \in C I R C(P, Q, V)(\varphi)$ iff any model of $\varphi$ minimal for $\prec_{(P, Q, V)}$ is a model of $\psi$.

This is the semantical definition of circumscription [Lifschitz, 1994], in the propositional case as given in [Moinard and Rolland, 1998b]. The set $\operatorname{CIRC}(P, Q, V)(\varphi)$ is equivalent to the formula having for models the models of $\varphi$ minimal for $\prec_{(P, Q, V)}$, and $C I R C(P, Q, V)(\varphi)$ will sometimes be identified with this formula.

We get the following result:

$$
\begin{aligned}
\text { Theorem } 5.2 \operatorname{Circ}(P, Q, V)(\varphi) \models \psi & \text { iff } \\
\varphi & \models \text { ForgetLitVar }\left(\varphi \wedge \psi, P^{-}, V\right) .
\end{aligned}
$$

This is in fact a rewriting of an already known result. Some technical indications are useful. in order to relate this result with the literature. First, this result improves the following result:

\section{Proposition 5.3 [Lang et al., 2003, Proposition 22]}

1. If $\varphi$ does not contain the propositional symbols in $V$, then $\operatorname{Circ}(P, Q, V)(\varphi) \models \psi$ iff $\varphi \models$ ForgetLit $\left(\varphi \wedge \psi, P^{-} \cup V^{ \pm}\right)$.

2. In the general case, $\quad \operatorname{Circ}(P, Q, V)(\varphi) \models \psi \quad$ iff $\varphi \models$ ForgetLit $\left(\varphi \wedge \neg\right.$ ForgetLit $\left.\left(\varphi \wedge \neg \psi, P^{-} \cup V^{ \pm}\right), P^{-} \cup V^{ \pm}\right)$.

Point 1 concerns circumscription without varying proposition since, if $\psi$ has no symbol in $V, \operatorname{Circ}(P, Q, V)(\varphi) \models \psi$ iff $\operatorname{Circ}(P, Q, \emptyset)(F \operatorname{Forget} V(\varphi, V)) \models \psi$.

As shown in [Lang et al., 2003], the two points of this result are respectively consequences of the two points of an older result:

\section{Proposition 5.4 [Przymusinski, 1989, Theorems 2.5 - 2.6]}

1. [Theorem 2.5] If $\psi$ is without symbol in $V$, then $\operatorname{Circ}(P, Q, V)(\varphi) \models \psi$ iff for any clause c made of literals not in $P^{-} \cup V^{ \pm}, \varphi \wedge \psi \models c$ implies $\varphi \models c$.
2. [Theorem 2.6] $\operatorname{Circ}(P, Q, V)(\varphi) \models \psi$ iff there exists $\gamma=$ ForgetLit $\left(\gamma^{\prime}, P^{-} \cup V^{ \pm}\right)$for some formula $\gamma^{\prime}$, such that $C I R C(P, Q, V)(\varphi) \models \neg \gamma$ and $\varphi \models \gamma \vee \psi$.

The second point is also a two stages method, which greatly increases its complexity: we must examine all the formulas $\gamma$ in a rather large set and check, by using the first point, whether $\neg \gamma$ is entailed by the circumscription or not. The first point is again in fact restricted to the circumscriptions without varying proposition.

Let us introduce here another nonmonotonic formalism, which encompasses the formalisms implicitly used in the first points of Propositions 5.3 and 5.4.

Definition 5.5 [Siegel and Forget, 1996] An X-mapping is a mapping $f_{X}: \mathbf{P L} \hookrightarrow \mathcal{P}(\mathbf{P L})$ defined from a set $X$ of formulas in PL as follows:

$$
\varphi \in f_{X}(\psi) \text { iff } T h(\psi \wedge \varphi) \cap X \subseteq T h(\psi) .
$$

This notion has been introduced in order to facilitate the computation of a nonmonotonic formalism. In fact, this notion appeared before the referenced paper, e.g. in [Suchenek, 1993] and even in earlier papers about the computation of minimization formalisms. The novelty in [Siegel and Forget, 1996] was a clear identification of the kind of formalism at hand, in particular it is shown there that, contrarily to circumscription and other formalisms, we do not get a theory: the set $f_{X}(\varphi)$ is generally not closed for $\wedge$, thus $f_{X}(\varphi)$ in the general case cannot be assimilated to a formula.

We can choose for $X$ any set having the same $\wedge$-closure (cf Remark 2.3).

Let $f$ be a mapping: $\mathbf{P L} \hookrightarrow \mathcal{P}(\mathbf{P L})$. The set of the formulas inaccessible for $f$ is the set of all the formulas $\psi$ for which there exist no formula $\varphi$ such that $\psi \in f(\varphi)$ and $\varphi \not \forall \psi$. It is known (and easy to check) that for any $\mathrm{X}$-mapping $f_{X}$, the set of all the inaccessible formulas is (up to logical equivalence) the $\wedge$-closure of $X$.

Points 1 in Propositions 5.3 and 5.4 can be written respectively as follows, where $\equiv \bar{V}$ means equivalent on the set of the formulas without symbol in $V$ :

- $\operatorname{Circ}(P, Q, V)(\varphi) \equiv \bar{V} f_{X}(\varphi)$ where $X$ is the set given in Proposition 4.4-1 [or alternatively any of the sets indicated in Proposition 4.4 (following)-1].

- $\operatorname{Circ}(P, Q, V)(\varphi) \equiv \bar{V} f_{X}(\varphi)$ where $X$ is the set of all the clauses with literals in $P^{+} \cup Q^{ \pm}$.

It is clear from Remark 2.3 and Proposition 4.4 that these two "Points 1" are equivalent, as shown in [Lang et al., 2003].

We will show now that, in Proposition 5.4 (the same considerations hold in Proposition 5.3) Point 2 is (almost) a direct consequence of Point 1 , when using an old result about the elimination of varying propositions in circumscription thanks to variable forgetting [Lifschitz, 1994, Proposition 


\subsection{1] (first published in 1985):}

$$
\begin{aligned}
& \operatorname{Circ}(P, Q, V)(\varphi) \models \psi \quad \text { iff } \\
& \operatorname{Circ}(P, Q, \emptyset)(\text { Forget }(\varphi, V)) \cup\{\varphi\} \models \psi
\end{aligned}
$$

Indeed, from $\left(\mathrm{Eq}_{L}\right)$, we get $\operatorname{Circ}(P, Q, V)(\varphi) \models \psi$ iff there exists a formula $\neg \gamma$ without symbol in $V$ such that $\operatorname{Circ}(P, Q, \emptyset)(F \operatorname{Frget} V(\varphi, V)) \models \neg \gamma$ and $\varphi \wedge \neg \gamma \models \psi$. Since $\neg \gamma$ is without symbol in $V$, this is equivalent to: $\operatorname{Circ}(P, Q, V)(\varphi) \models \neg \gamma$ and $\varphi \models \gamma \vee \psi$. The only difference is that Przymusinski has improved this result a bit by restricting the set of the available formulas for $\gamma$, since $P^{-}$can be suppressed ("forgotten") also, and not only $V$.

The connexions between X-logic and circumscription are now well known [Suchenek, 1993; Siegel and Forget, 1996; Moinard and Rolland, 1998a]. It is convenient here to introduce a few technical notions.

Definition 5.6 Let us call positive in $\prec_{(P, Q, V)}$ any formula $\varphi$ whose set of models is finishing for $\prec_{(P, Q, V)}$ : if $\mu \models \varphi$ and $\mu \prec_{(P, Q, V)} \nu$, then $\nu \models \varphi$.

Directly from Definition 4.1 we get that ForgetLit $\operatorname{Var}(\varphi, P, V)$ is positive in $\prec_{(P, Q, V)}$.

Notice that the "converse" also holds since each $\varphi$ positive in $\prec_{(P, Q, V)}$ is equal to Forget LitVar $(\varphi, P, V)$.

Example 2 (Example 1 following) With $\varphi, P, V, Q$ as in Example 1, we get clearly $\operatorname{Mod}\left(\right.$ ForgetLitVar $\left.\left(\varphi, P^{-}, V\right)\right)=$ $\left\{\nu \in \operatorname{Mod} / \mu \prec_{(P, Q, V)} \nu\right.$ or $\mu=\nu$ for some $\mu \in$ $\operatorname{Mod}(\varphi)\}$. This set is clearly finishing for $\prec(P, Q, V)$.

With these technical indications, it would be easy to complete a direct proof of Theorem 5.2. However, a new proof is not necessary since Theorem 5.2 is an immediate consequence of Proposition 4.4 together with the following already known result:

Proposition 5.7 [Moinard and Rolland, 1998b, Theorem 6.40] $\operatorname{Circ}(P, Q, V)=f_{X}$ for any set $X$ which has (up to logical equivalence) for its $\wedge$-closure the set of the formulas positive in $\prec(P, Q, V)$.

Let us provide an example in order to show how Theorem 5.2 works in practice.

Example $3 P, Q, V$ are as in Example 1.

$\varphi^{\prime}=(b \wedge c) \vee(a \wedge \neg b \wedge \neg c \wedge \neg d)$ and

$\psi^{\prime}=(\neg a \wedge b) \vee(a \wedge \neg b \wedge \neg c \wedge \neg d) \vee(\neg a \wedge c \wedge d)$.

We get that $\varphi^{\prime} \wedge \psi^{\prime}$ is equivalent to $\varphi$ of Example 1 .

Since we have $\varphi^{\prime} \models\left(\right.$ ForgetLitVar $\left.\left(\varphi, P^{-}, V\right)\right)$, we conclude that we have $\operatorname{Circ}(P, Q, V)\left(\varphi^{\prime}\right) \models \psi^{\prime}$.

The ForgetLitVar approach provides new insights into the effective methods for computing circumscriptions: In particular, it provides single stage process, instead of the two stages process of Propositions 5.3 and 5.4. It provides also a concrete way for computing circumscription thanks to Proposition 5.7, giving some flesh to the method for computing circumscription from their inaccessible formulas which has been described in [Moinard and Rolland, 1998a; 1998b].

\section{Conclusion and perspectives}

We have provided the semantical and several syntactical characterizations for a new notion, extending the notion of literal forgetting introduced in [Lang et al., 2003] to the cases where some propositional symbols are allowed to vary. These results show that the new notion is not significantly harder than literal forgetting without varying symbols. The various characterizations provide various effective ways for computing the results, depending on the form in which the formulas appear.

Then, we have developed an application for computing circumscriptions. Doing this, we have reinforced the connexions (already found in [Lang et al., 2003]) between the literal forgetting method and an older method from [Przymusinski, 1989]. Our work has allowed to transform the two stages methods which were known, either by explicit literal forgetting or by Przymunsinski's way, into simpler one stage methods. Doing so, we have shown that the literal forgetting method (thus also Przymunsinski's method) is strongly related to the method of X-mappings. These connexions provide a simpler presentation of various results, including the simplification (evoked above) of Przymunsinski's method for computing circumscription.

Notice that the "inaccessible formulas" of some non classical inference mechanism (built upon classical logic) have a clear meaning from the knowledge representation point of view. These formulas are the formulas which cannot be obtained as a new result (meaning not already obtained by classical inference) by using the inference mechanism. Thus, the description, provided here, of the way of computing circumscription from its inaccessible formulas, in terms of literal forgetting, provides new insights from the common sense reasoning point of view.

Also, the method described here could pave the way for more applications of the "forget literal method" towards already known formalisms. As written in [Lang et al., 2003], results similar to the ones given here could be given for other closed world formalisms. Also, some of the various formalisms evoked in [Lang et al., 2003, p. 413] could take profit from the introduction of varying propositional symbols. Moreover, since X-mappings can falsify the stability for $\wedge$ (in the default terminology, several extensions are possible), further generalizations of the notion of literal forgetting could possibly deal with some nonmonotonic formalisms falsifying the stability for $\wedge$.

An interest of the method of literal forgetting, since it consists in small and easy manipulations of propositional formulas, is that it can help the effective computation. As shown in [Lang et al., 2003], we cannot hope that this will solve all the problems, but it should help in providing significant practical 
improvements.

\section{References}

[Lang et al., 2003] Jerome Lang, Paolo Liberatore, and Pierre Marquis. Propositional Independence - FormulaVariable Independence and Forgetting. (Electronic) Journal of Artificial Intelligence Research, 18:391-443, 2003. http: //WWW. JAIR. ORG/.

[Lifschitz, 1994] Vladimir Lifschitz. Circumscription. In Dov M. Gabbay, C.J. Hogger, and J.A. Robinson, editors, Handbook of Logic in Artificial Intelligence and Logic Programming, Volume 3: Non-Monotonic and Uncertainty Reasoning, pages 297-352. Oxford University Press, 1994.

[McCarthy, 1986] John McCarthy. Application of circumscription to formalizing common sense knowledge. Artificial Intelligence, 28(1):89-116, February 1986.

[Moinard and Rolland, 1998a] Yves Moinard and Raymond Rolland. Circumscriptions from what they cannot do (Preliminary report). In Working papers of Common Sense'98, pages 20-41, London, January 1998.

http://www.ida.liu.se/ext/etai/nj/fcs-98/.

[Moinard and Rolland, 1998b] Yves Moinard and Raymond Rolland. Propositional circumscriptions. Technical report, INRIA, Research Report RR-3538, Rennes, France, October 1998.

[Przymusinski, 1989] Teodor C. Przymusinski. An Algorithm to Compute Circumscription. Artificial Intelligence, 38(1):49-73, February 1989.

[Siegel and Forget, 1996] Pierre Siegel and Lionel Forget. A representation theorem for preferential logics. In Luigia Carlucci Aiello, Jon Doyle, and Stuart Shapiro, editors, KR'96, pages 453-460, Cambridge, November 1996. Morgan Kaufmann.

[Suchenek, 1993] Marek A. Suchenek. First-Order Syntactic Characterizations of Minimal Entailment, DomainMinimal Entailment, and Herbrand Entailment. Journal of Automated reasoning, 10:237-263, 1993. 